\title{
Approximation on a class of Phillips operators generated by $q$-analogue
}

\section{Abdullah Alotaibi ${ }^{*}$}

\section{"Correspondence:}

mathker11@hotmail.com

${ }^{1}$ Operator Theory and Applications

Research Group, Department of

Mathematics, Faculty of Science,

King Abdulaziz University, Jeddah,

Saudi Arabia

\section{Springer}

\begin{abstract}
The main purpose of this article is to introduce a new generalization of q-Phillips operators generated by Dunkl exponential function. We establish some approximation results for these operators. We also determine the order of approximation, and the rate of convergence in terms of the modulus of continuity of order one and two. Moreover, we obtain some direct theorems.
\end{abstract}

MSC: Primary 41A25; 41A36; secondary 33C45

Keywords: Bernstein operator; Szász operator; q-Phillips operator; Dunkl analogue; Modulus of continuity; Weighted modulus of continuity

\section{Introduction}

Bernstein polynomials play a very important role in approximation process. For a positive integer $n \geqq 1$ and a function $g$ defined on $[0,1]$, Bernstein defined the positive linear operators $B_{n}: C[0,1] \rightarrow C[0,1]$ by

$$
B_{n}(g ; x)=\sum_{k=0}^{n}\left(\begin{array}{l}
n \\
k
\end{array}\right) x^{k}(1-x)^{n-k} g\left(\frac{k}{n}\right), \quad x \in[0,1] .
$$

For some recent work on Bernstein operators, we refer to [21, 26, 27, 32, 46]. In 1950, Szász defined the operators [48]

$$
S_{m}(g ; x)=e^{-m x} \sum_{k=0}^{\infty} \frac{(m x)^{k}}{k !} g\left(\frac{k}{m}\right) \quad(x \in[0, \infty), m \in \mathbb{N})
$$

for a continuous function $g$ on $[0, \infty)$. The construction of Szász type operators is accomplished, by a newly parameter $\kappa \geqq 0$, and it is known as the Dunkl generalization. It was given by Sucu [47] with the help of [43]. The $q$-Hermite type polynomials were introduced by Cheikh et al. [13] by applying a new parameter $\kappa>-\frac{1}{2}$. The exponential functions and recursion formula on the Dunkl generalization are given by

$$
e_{\kappa, q}(x)=\sum_{m=0}^{\infty} \frac{x^{m}}{\gamma_{\kappa, q}(m)}, \quad \text { and } \quad E_{\kappa, q}(x)=\sum_{m=0}^{\infty} \frac{q^{\frac{m(m-1)}{2}} x^{m}}{\gamma_{\kappa, q}(m)},
$$

(c) The Author(s) 2020. This article is licensed under a Creative Commons Attribution 4.0 International License, which permits use, sharing, adaptation, distribution and reproduction in any medium or format, as long as you give appropriate credit to the original author(s) and the source, provide a link to the Creative Commons licence, and indicate if changes were made. The images or other third party material in this article are included in the article's Creative Commons licence, unless indicated otherwise in a credit line to the material. If material is not included in the article's Creative Commons licence and your intended use is not permitted by statutory regulation or exceeds the permitted use, you will need to obtain permission directly from the copyright holder. To view a copy of this licence, visit http://creativecommons.org/licenses/by/4.0/. 


$$
\begin{aligned}
& \gamma_{\kappa, q}(m+1)=\left(\frac{1-q^{2 \kappa \theta_{m+1}+m+1}}{1-q}\right) \gamma_{\kappa, q}(m), \quad m \in \mathbb{N}, \\
& \theta_{m}= \begin{cases}0 & \text { if } m=2,4,6, \ldots \\
1 & \text { if } m=1,3,5, \ldots\end{cases}
\end{aligned}
$$

We recall the basic information regarding the $q$-calculus:

$$
\begin{gathered}
{[n]_{q}= \begin{cases}\frac{1-q^{n}}{1-q} & \text { for } q \neq 1, n \in \mathbb{N}, \\
1 & \text { for } q=1, \\
0 & \text { for } n=0,\end{cases} } \\
{[n]_{q} != \begin{cases}1 & \text { for } n=0, \\
\prod_{k=1}^{n}[k]_{q} & \text { for } n \in \mathbb{N} .\end{cases} }
\end{gathered}
$$

are the $q$-integer $[n]_{q}$ and $q$-factorial $[n]_{q}$ !, respectively. İçöz and Çekim [17] wrote the Szász operators as follows:

$$
D_{m, q}(g ; x)=\frac{1}{e_{\kappa, q}\left([m]_{q} x\right)} \sum_{k=0}^{\infty} \frac{\left([m]_{q} x\right)^{k}}{\gamma_{\kappa, q}(k)} g\left(\frac{1-q^{2 \kappa \theta_{k}+k}}{1-q^{m}}\right) .
$$

Recently, the Szász operators have many improvements and modifications in approximation process (see $[1,24,25,35,42])$. The $q$-analogue of some other interesting operators has been studied in $[2,37,45,49]$ and the references therein. An additional approach to improving the quantum calculus is post-quantum calculus via these types of generalizations; it was proposed in $[3-5,7,8,18,23,28,33,34,36]$ (see also [39, 40]).

In this manuscript, we emphasize a new generalization of $q$-Phillips operators by introducing the new parameters and increasing and unbounded sequences of positive numbers. For more details of the approximation to classical Phillips operators via the Dunkl type version, see the recent article [38]. We study the convergence results in modulus of continuity of order one and two. Moreover, we investigate the rate of convergence for functions belonging to the Lipschitz class and also prove some direct theorems. For further information and the results used in this article we mention here some related articles (see $[6,19,22,44])$.

\section{Operators and their associated moments}

Let $\left\{\alpha_{[m]_{q}}\right\}_{m \geqq 1}$ and $\left\{\beta_{[m]_{q}}\right\}_{m \geqq 1}$ be the increasing and unbounded sequences of positive numbers such that

$$
\lim _{m \rightarrow \infty} \frac{1}{\beta_{[m]_{q}}} \rightarrow 0 \text { and } \quad \frac{\alpha_{[m]_{q}}}{\beta_{[m]_{q}}}=1+O\left(\frac{1}{\beta_{[m]_{q}}}\right)
$$

For $m=1,2, \ldots$, we denote the nodes $\nabla_{m}$ by

$$
\nabla_{m}=m+2 \kappa \theta_{m}, \quad \kappa \geq-\frac{1}{2}
$$


For all $x \in[0, \infty), n \in \mathbb{N} \cup\{0\}$ and every $g \in C_{\eta}[0, \infty)=\left\{f \in C[0, \infty): g(t)=O\left(t^{\eta}\right), t \rightarrow\right.$ $\infty$ ) with $\eta>n$, we define

$$
\begin{aligned}
& \mathcal{S}_{m, q}^{*}\left(g ; \alpha_{[m]_{q}}, \beta_{[m]_{q}} ; x\right) \\
& \quad=\frac{\alpha_{[m]_{q}}}{e_{\kappa, q}\left(\alpha_{[m]_{q}} x\right)} \sum_{j=0}^{\infty} \mathcal{T}_{m, q}^{\kappa}(x) \int_{0}^{\infty / 1-q} \frac{e_{\kappa, q}\left(-\alpha_{[m]_{q}} t\right)\left(\alpha_{[m]_{q}} t\right)^{\nabla_{j}}}{\left[\nabla_{j}\right]_{q} !} f\left(q^{\nabla_{j}} \frac{\alpha_{[m]_{q}}}{\beta_{[m]_{q}}} t\right) \mathrm{d}_{q} t
\end{aligned}
$$

where

$$
\mathcal{T}_{m, q}^{\kappa}(x)=\frac{\left(\alpha_{[m]_{q}} x\right)^{j}}{\gamma_{\kappa, q}(j)} q^{\frac{\left(\nabla_{j}\right)\left(\nabla_{j}+1\right)}{2}}
$$

Definition 2.1 For all $m>0$ and $q \in(0,1)$, the generalized $q$-Gamma function is defined by

$$
\begin{aligned}
& \Gamma_{q}(m)=\int_{0}^{1 / 1-q} x^{m-1} E_{q}(-q x) \mathrm{d}_{q} x, \quad m>0, \\
& \gamma_{q}^{K}(m)=\int_{0}^{\infty / K(1-q)} x^{m-1} e_{q}(-x) \mathrm{d}_{q} x, \quad m>0,
\end{aligned}
$$

where $\Gamma_{q}(m)=L(K ; m) \gamma_{q}^{K}(m)$ and $L(K ; m)=\frac{1}{1+K} K^{m}\left(1+\frac{1}{K}\right)_{q}^{m}(1+K)_{q}^{m-1}$. Moreover, in particular for any positive integer $m$ we have $L(K ; m)=q^{\frac{m(m-1)}{2}}$ and $\Gamma_{q}(m)=q^{\frac{m(m-1)}{2}} \gamma_{q}^{K}(m)$, which also satisfies the following equation:

$$
\Gamma_{q}(m+1)= \begin{cases}{[m]_{q} \Gamma_{q}(m)} & \text { for } m>0 \\ 1 & \text { for } m=0\end{cases}
$$

For more details, see [15].

Lemma 2.2 Let $\mathcal{S}_{m, q}^{*}(\cdot ; \cdot)$ be the operators defined by (2.3). Then we have:

(1) $\mathcal{S}_{m, q}^{*}\left(1 ; \alpha_{[m]_{q}}, \beta_{[m]_{q}} ; x\right)=1$,

(2) $\mathcal{S}_{m, q}^{*}\left(t ; \alpha_{[m]_{q}}, \beta_{[m]_{q}} ; x\right)=\left(\frac{\alpha_{[m]_{q}}}{\beta_{[m]_{q}}}\right) x+\frac{1}{q \beta_{[m]_{q}}}$,

(3) $\mathcal{S}_{m, q}^{*}\left(t^{2} ; \alpha_{[m]_{q}}, \beta_{[m]_{q}} ; x\right) \leqq \frac{(1+q)}{q^{3}\left(\beta_{[m]_{q}}\right)^{2}}+\frac{\alpha_{[m]_{q}}}{\left(\beta_{[m]_{q}}\right)^{2}}\left(\frac{1+2 q}{q^{2}}+[1+2 \kappa]_{q}\right) x$

$$
+\left(\frac{\alpha_{[m]_{q}}}{\beta_{[m]_{q}}}\right)^{2} x^{2}
$$

(4) $\mathcal{S}_{m, q}^{*}\left(t^{3} ; \alpha_{[m]_{q}}, \beta_{[m]_{q}} ; x\right) \leqq \frac{(1+q)\left(1+q+q^{2}\right)}{q^{6}\left(\beta_{[m]_{q}}\right)^{3}}$

$$
\begin{aligned}
& +\frac{\alpha_{[m]_{q}}}{q^{5}\left(\beta_{[m]_{q}}\right)^{3}}\left\{\left(1+3 q+4 q^{2}+3 q^{3}\right)\right. \\
& \left.+q^{2}\left(1+2 q+3 q^{2}\right)[1+2 \kappa]_{q}+q^{5}[1+2 \kappa]_{q}^{2}\right\} x
\end{aligned}
$$




$$
\begin{aligned}
& +\frac{\alpha_{[m]_{q}}}{q^{4}\left(\beta_{[m]_{q}}\right)^{3}}\left\{q\left(1+2 q+3 q^{2}\right)+3 q^{4}[1+2 \kappa]_{q}\right\} x^{2} \\
& +\left(\frac{\alpha_{[m]_{q}}}{\beta_{[m]_{q}}}\right)^{3} x^{3}
\end{aligned}
$$

(5) $\mathcal{S}_{m, q}^{*}\left(t^{4} ; \alpha_{[m] q}, \beta_{[m] q} ; x\right) \leqq \frac{(1+q)\left(1+2 q+3 q^{2}+3 q^{3}+2 q^{4}+q^{5}\right)}{q^{10}\left(\beta_{[m] q}\right)^{4}}$

$$
\begin{aligned}
& +\frac{\alpha_{[m]_{q}}}{q^{9}\left(\beta_{[m]_{q}}\right)^{4}}\left\{\left(1+4 q+8 q^{2}+12 q^{3}+12 q^{4}+9 q^{5}+4 q^{6}\right)\right. \\
& +q^{2}\left(1+3 q+7 q^{2}+9 q^{3}+9 q^{4}+6 q^{5}\right)[1+2 \kappa]_{q} \\
& \left.+q^{5}\left(1+2 q+3 q^{2}+4 q^{3}\right)[1+2 \kappa]_{q}^{2}+q^{9}[1+2 \kappa]_{q}^{3}\right\} x \\
& +\frac{\left(\alpha_{\left.[m]_{q}\right)^{2}}\right.}{q^{8}\left(\beta_{[m]_{q}}\right)^{4}}\left\{q\left(1+3 q+7 q^{2}+9 q^{3}+9 q^{4}+6 q^{5}\right)\right. \\
& \left.+q^{4}\left(1+2 q+3 q^{2}+4 q^{3}\right)[1+2 \kappa]_{q}+7 q^{8}[1+2 \kappa]_{q}^{2}\right\} x^{2} \\
& +\frac{\left(\alpha_{[m]_{q}}\right)^{3}}{q^{7}\left(\beta_{[m]_{q}}\right)^{4}}\left\{q^{3}\left(1+2 q+3 q^{2}+4 q^{3}\right)+6 q^{7}[1+2 \kappa]_{q}\right\} x^{3} \\
& +\left(\frac{\alpha_{[m]_{q}}}{\beta_{[m]_{q}}}\right)^{4} x^{4} .
\end{aligned}
$$

Proof From the generalized $q$-Gamma function defined by Definition 2.1, we see that

$$
\begin{aligned}
& \int_{0}^{\infty / 1-q} q^{\frac{\left(\nabla_{j}\right)\left(\nabla_{j}+1\right)}{2}} \frac{e_{\kappa, q}\left(-\alpha_{[m]_{q}} t\right)\left(\alpha_{[m]_{q}} t\right)^{\nabla_{j}}}{\left[\nabla_{j}\right]_{q} !}\left(q^{\nabla_{j}} \frac{\alpha_{[m]_{q}}}{\beta_{[m]_{q}}} t\right)^{u} \mathrm{~d}_{q} t \\
& =\frac{1}{\alpha_{[m]_{q}}\left(\beta_{[m]_{q}}\right)^{u}} \frac{1}{\left[\nabla_{j}\right]_{q} !} q^{\frac{\left(\nabla_{j}\right)\left(\nabla_{j}+1\right)}{2}+u\left(\nabla_{j}\right)} \int_{0}^{\infty / 1-q}\left(\alpha_{[m]_{q}} t\right)^{\nabla_{j}+u} e_{\kappa, q}\left(-\alpha_{[m]_{q}} t\right) \alpha_{[m]_{q}} \mathrm{~d}_{q} t \\
& =\frac{1}{\alpha_{[m]_{q}}\left(\beta_{\left.[m]_{q}\right)^{u}}\right.} \frac{1}{\left[\nabla_{j}\right]_{q} !} q^{\frac{\left(\nabla_{j}\right)\left(\nabla_{j}+1\right)}{2}+u\left(\nabla_{j}\right)} \int_{0}^{\infty / 1-q} t^{\nabla_{j}+u} e_{\kappa, q}(-t) \mathrm{d}_{q} t \\
& =\frac{1}{\alpha_{[m]_{q}}\left(\beta_{\left.[m]_{q}\right)^{u}} t\right.} \frac{1}{\left[\nabla_{j}\right]_{q} !} q^{\frac{\left(\nabla_{j}\right)\left(\nabla_{j}+1\right)}{2}+u\left(\nabla_{j}\right)} \gamma_{q}^{1}\left(\nabla_{j}+u+1\right) \\
& =\frac{1}{\alpha_{[m]_{q}}\left(\beta_{\left.[m]_{q}\right)^{u}} u\right.} \frac{1}{\left[\nabla_{j}\right]_{q} !} q^{\frac{\left(\nabla_{j}\right)\left(\nabla_{j}+1\right)}{2}+u\left(\nabla_{j}\right)} \frac{\left[\nabla_{j}+u\right]_{q} !}{\alpha_{[m]_{q}}\left(\beta_{\left.[m]_{q}\right)^{u}} \frac{\left[\nabla_{j}+u\right]_{q} !}{\left[\nabla_{j}\right]_{q} !} \frac{1}{q^{\frac{u(u+1)}{2}}} .\right.} q^{\frac{\left(\nabla_{j}+u\right)\left(\nabla_{j}+u+1\right)}{2}}
\end{aligned}
$$

If $u=0$ then $g(t)=1$, and hence

$$
\mathcal{S}_{m, q}^{*}\left(1 ; \alpha_{[m]_{q}}, \beta_{[m]_{q}} ; x\right)=\frac{\alpha_{[m]_{q}}}{e_{\kappa, q}\left(\alpha_{[m]_{q}} x\right)} \sum_{j=0}^{\infty} \frac{\left(\alpha_{[m]_{q}} x\right)^{j}}{\gamma_{\kappa, q}(j)} \frac{\left[\nabla_{j}\right]_{q} !}{\alpha_{[m]_{q}}\left[\nabla_{j}\right]_{q} !}
$$

$=1$. 
If $u=1$, then $g(t)=t$, hence,

$$
\begin{aligned}
\mathcal{S}_{m, q}^{*}\left(t ; \alpha_{[m]_{q}}, \beta_{[m]_{q}} ; x\right)= & \frac{\left(\alpha_{[m]_{q}}\right)^{2}}{\beta_{[m]_{q}} e_{\kappa, q}\left(\alpha_{[m]_{q}} x\right)} \sum_{j=0}^{\infty} \frac{\left(\alpha_{[m]_{q}} x\right)^{j}}{\gamma_{\kappa, q}(j)} \frac{\left[\nabla_{j}+1\right]_{q} !}{q\left(\alpha_{[m]_{q}}\right)^{2}\left[\nabla_{j}\right]_{q} !} \\
= & \frac{1}{q \beta_{[m]_{q}} e_{\kappa, q}\left(\alpha_{[m]_{q}} x\right)} \sum_{j=0}^{\infty} \frac{\left(\alpha_{[m]_{q}} x\right)^{j}}{\gamma_{\kappa, q}(j)}\left[\nabla_{j}+1\right]_{q} \\
= & \frac{1}{q \beta_{[m]_{q}} e_{\kappa, q}\left(\alpha_{[m]_{q}} x\right)} \sum_{j=0}^{\infty} \frac{\left(\alpha_{[m]_{q}} x\right)^{j}}{\gamma_{\kappa, q}(j)} \\
& +\frac{1}{\beta_{[m]_{q}} e_{\kappa, q}\left(\alpha_{[m]_{q}} x\right)} \sum_{j=0}^{\infty} \frac{\left(\alpha_{[m]_{q}} x\right)^{j}}{\gamma_{\kappa, q}(j)}\left[\nabla_{j}\right]_{q} \\
= & \left(\frac{\alpha_{[m]_{q}}}{\beta_{[m]_{q}}}\right) x+\frac{1}{q \beta_{[m]_{q}}} .
\end{aligned}
$$

Take $u=2$, then, for $g(t)=t^{2}$, we have

$$
\begin{aligned}
\mathcal{S}_{m, q}^{*}\left(t^{2} ; \alpha_{[m]_{q}}, \beta_{[m]_{q}} ; x\right)= & \frac{\left(\alpha_{[m]_{q}}\right)^{3}}{\left(\beta_{[m]_{q}}\right)^{2} e_{\kappa, q}\left(\alpha_{[m]_{q}} x\right)} \sum_{j=0}^{\infty} \frac{\left(\alpha_{[m]_{q}} x\right)^{j}}{\gamma_{\kappa, q}(j)} \frac{\left[\nabla_{j}+2\right]_{q} !}{q^{3}\left(\alpha_{[m]_{q}}\right)^{3}\left[\nabla_{j}\right]_{q} !} \\
= & \frac{1}{q^{3}\left(\beta_{[m]_{q}}\right)^{2} e_{\kappa, q}\left(\alpha_{[m]_{q}} x\right)} \sum_{j=0}^{\infty} \frac{\left(\alpha_{[m]_{q}} x\right)^{j}}{\gamma_{\kappa, q}(j)}\left[\nabla_{j}+2\right]_{q}\left[\nabla_{j}+1\right]_{q} \\
= & \frac{1}{q^{3}\left(\beta_{[m]_{q}}\right)^{2} e_{\kappa, q}\left(\alpha_{[m]_{q}} x\right)} \sum_{j=0}^{\infty} \frac{\left(\alpha_{[m]_{q}} x\right)^{j}}{\gamma_{\kappa, q}(j)} \\
= & \frac{(1+q)}{q^{3}\left(\beta_{[m]_{q}}\right)^{2}}+\frac{\alpha_{[m]_{q}}(1+2 q)}{q^{2}\left(\beta_{[m]_{q}}\right)^{2}} x \\
& +\frac{1}{\left(\beta_{[m]_{q}}\right)^{2} e_{\kappa, q}\left(\alpha_{[m]_{q}} x\right)} \sum_{j=0}^{\infty} \frac{\left(\alpha_{[m]_{q}} x\right)^{j}}{\gamma_{\kappa, q}(j)}\left[\nabla_{j}\right]_{q}^{2} .
\end{aligned}
$$

From [17] and by (1.7), we use

$$
\begin{aligned}
& \frac{1}{e_{\kappa, q}\left(\alpha_{[m]_{q}} x\right)} \sum_{j=0}^{\infty} \frac{\left(\alpha_{[m]_{q}} x\right)^{k}}{\gamma_{\kappa, q}(j)}\left[\nabla_{j}\right]_{q}^{2} \geq\left(\alpha_{[m]_{q}} x\right)^{2}+q^{2 \kappa}[1-2 \kappa]_{q} \frac{e_{\kappa, q}\left(q \alpha_{[m]_{q}} x\right)}{e_{\kappa, q}\left(\alpha_{[m]_{q}} x\right)} \alpha_{[m]_{q}} x, \\
& \frac{1}{e_{\kappa, q}\left(\alpha_{[m]_{q}} x\right)} \sum_{j=0}^{\infty} \frac{\left(\alpha_{[m]_{q}} x\right)^{k}}{\gamma_{\kappa, q}(j)}\left[\nabla_{j}\right]_{q}^{2} \leq\left(\alpha_{[m]_{q}} x\right)^{2}+[1+2 \kappa]_{q} \alpha_{[m]_{q}} x .
\end{aligned}
$$

For $u=3, g(t)=t^{3}$ and for $u=4, g(t)=t^{4}$, we get

$$
\mathcal{S}_{m, q}^{*}\left(t^{3} ; \alpha_{[m]_{q}}, \beta_{[m]_{q}} ; x\right)=\frac{1}{q^{4}\left(\beta_{[m]_{q}}\right)^{3} e_{\kappa, q}\left(\alpha_{[m]_{q}} x\right)} \sum_{j=0}^{\infty} \frac{\left(\alpha_{[m]_{q}} x\right)^{j}}{\gamma_{\kappa, q}(j)}\left[\nabla_{j}+3\right]_{q}\left[\nabla_{j}+2\right]_{q}\left[\nabla_{j}+1\right]_{q}
$$


and

$$
\begin{aligned}
\mathcal{S}_{m, q}^{*}\left(t^{4} ; \alpha_{[m]_{q}}, \beta_{[m] q} ; x\right)= & \frac{1}{q^{10}\left(\beta_{[m] q}\right)^{4} e_{\kappa, q}\left(\alpha_{[m]_{q}} x\right)} \sum_{j=0}^{\infty} \frac{\left(\alpha_{[m]_{q}} x\right)^{j}}{\gamma_{\kappa, q}(j)} \\
& \times\left[\nabla_{j}+4\right]_{q}\left[\nabla_{j}+3\right]_{q}\left[\nabla_{j}+2\right]_{q}\left[\nabla_{j}+1\right]_{q} .
\end{aligned}
$$

From [37] we know that

$$
\begin{aligned}
{\left[\nabla_{j}+\right.} & 3]_{q}\left[\nabla_{j}+2\right]_{q}\left[\nabla_{j}+1\right]_{q} \\
= & (1+q)\left(1+q+q^{2}\right)+\left\{q(1+2 q)\left(1+q+q^{2}\right)+q^{3}(1+q)\right\}\left[\nabla_{j}\right]_{q} \\
& +\left\{q^{3}\left(1+q+q^{2}\right)+q^{4}(1+2 q)\right\}\left[\nabla_{j}\right]_{q}^{2}+q^{6}\left[\nabla_{j}\right]_{q}^{3}, \\
{\left[\nabla_{j}+\right.} & 4]_{q}\left[\nabla_{j}+3\right]_{q}\left[k+2 \kappa \theta_{k}+2\right]_{q}\left[\nabla_{j}+1\right]_{q} \\
= & (1+q)\left(1+2 q+3 q^{2}+3 q^{3}+2 q^{4}+q^{5}\right)+\left\{q(1+2 q)\left(1+2 q+3 q^{2}+3 q^{3}+2 q^{4}+q^{5}\right)\right. \\
& \left.+q^{3}(1+q)\left(1+2 q+2 q^{2}+2 q^{3}\right)\right\}\left[\nabla_{j}\right]_{q} \\
& +\left\{q^{3}\left(1+2 q+3 q^{2}+3 q^{3}+2 q^{4}+q^{5}\right)+q^{4}(1+2 q)\left(1+2 q+2 q^{2}+2 q^{3}\right)\right. \\
& \left.+q^{7}(1+q)\right\}\left[\nabla_{j}\right]_{q}^{2} \\
& +\left\{q^{6}\left(1+2 q+2 q^{2}+2 q^{3}\right)+q^{8}(1+2 q)\right\}\left[\nabla_{j}\right]_{q}^{3}+q^{10}\left[\nabla_{j}\right]_{q}^{4} .
\end{aligned}
$$

Clearly by $D_{m, q}(f ; x)$ in [17]) and from [37] for $g(t)=t^{3}$ and $g(t)=t^{4}$ we get the result.

Lemma 2.3 Take $\mho_{j}=(t-x)^{j}$ for $j=1,2,3,4$ and $\delta_{m, q}=\sqrt{\mathcal{S}_{m, q}^{*}\left(\mho_{j} ; \alpha_{[m]_{q}}, \beta_{[m]_{q}} ; x\right)}$. Let $\mathcal{S}_{m, q}^{*}(\cdot ; \cdot)$ be the operators defined by (2.3). Then, for all $x \in[0, \infty)$ and $0<q<1$, we have $\mathcal{S}_{m, q}^{*}\left(\mho_{1} ; \alpha_{[m]_{q}}, \beta_{[m]_{q}} ; x\right)=\left(\frac{\alpha_{[m]_{q}}}{\beta_{[m]_{q}}}-1\right) x+\frac{1}{q \beta_{[m]_{q}}}$ and

$$
\left(\delta_{m, q}\right)^{2}=\left\{\begin{array}{l}
\frac{(1+q)}{q^{3}\left(\beta_{[m] q}\right)^{2}}+\frac{\alpha_{[m] q}}{\left(q \beta_{[m]_{q}}\right)^{2}}\left(1+2 q+q^{2}[1+2 \kappa]_{q}-2 q \beta_{[m]_{q}}\right) x+\left(\frac{\alpha_{[m] q}}{\beta_{[m]_{q}}}-1\right)^{2} x^{2} \\
\quad \text { or } j=2 \\
\frac{(1+q)^{2}}{q^{6}\left(\beta_{[m] q}\right)^{4}}+\frac{2(1+q) \alpha_{[m] q}}{\left(q^{5} \beta_{[m]_{q}}\right)^{4}}\left(1+2 q+q^{2}[1+2 \kappa]_{q}-2 q \beta_{[m]_{q}}\right) x \\
\quad+\left[\frac{\left(\alpha_{[m] q}\right)^{2}}{\left(q \beta_{[m] q}\right)^{4}}\left(1+2 q+q^{2}[1+2 \kappa]_{q}-2 q \beta_{[m]_{q}}\right)^{2}+\frac{2(1+q)}{q^{3}\left(\beta_{[m] q}\right)^{2}}\left(\frac{\alpha_{[m] q}}{\beta_{[m]_{q}}}-1\right)^{2}\right] x^{2} \\
\quad+\frac{2 \alpha_{[m] q}}{\left(q \beta_{[m]_{q}}\right)^{2}}\left(1+2 q+q^{2}[1+2 \kappa]_{q}-2 q \beta_{[m]_{q}}\right)\left(\frac{\alpha_{[m]_{q}}}{\beta_{[m]_{q}}}-1\right)^{2} x^{3}+\left(\frac{\alpha_{[m] q}}{\beta_{[m]_{q}}}-1\right)^{4} x^{4} \\
\text { for } j=4 .
\end{array}\right.
$$

\section{Approximation by Korovkin's theorem}

Korovkin's theorem has many applications and useful connections between the branches of mathematics and classical approximation theory (see [9]). In a very general context it is possible to define the Korovkin theorem presented in [9], so that it can be used in applications for the best approximation. Now we approximate the operators $\mathcal{S}_{m, q_{m}}^{*}(\cdot ; \cdot)$ by using Korovkin's theorem. Let $q=q_{m}$ with $q_{m} \in(0,1)$ and let $c$ be a fixed positive constant such that

$$
\lim _{n \rightarrow \infty} q_{m}=1 \text { and } \quad \lim _{n \rightarrow \infty} q_{m}^{n}=c .
$$


Theorem 3.1 Let $\left\{\alpha_{m_{q_{m}}}\right\}_{m \geqq 1}$ and $\left\{\beta_{m_{q_{m}}}\right\}_{m \geqq 1}$ be the sequences satisfying (3.1). Then, for every function $g$ such that $\left\{g: g \in C[0, \infty) \cap x \in[0, \infty)\right.$, and $\frac{g(x)}{1+x^{2}}$ is finite when $\left.x \rightarrow \infty\right\}$,

$$
\lim _{m \rightarrow \infty} \mathcal{S}_{m, q_{m}}^{*}\left(g ; \alpha_{[m]_{q_{m}}}, \beta_{[m]_{q_{m}}} ; x\right)=g(x)
$$

uniformly on each compact subset of $[0, \infty)$.

Proof The well-known Korovkin theorem implies that

$$
\lim _{m \rightarrow \infty} \mathcal{S}_{m, q_{m}}^{*}\left(t^{i} ; \alpha_{[m]_{q m}}, \beta_{[m]_{q_{m}}} ; x\right)=x^{i}, \quad i=0,1,2
$$

Clearly, from (2.1) and (3.1), we see that

$$
\lim _{m \rightarrow \infty} \mathcal{S}_{m, q_{m}}^{*}\left(t ; \alpha_{[m]_{q_{m}}}, \beta_{[m]_{q m}} ; x\right)=x, \quad \lim _{m \rightarrow \infty} \mathcal{S}_{m, q_{m}}^{*}\left(t^{2} ; \alpha_{[m]_{q_{m}}}, \beta_{[m]_{q_{m}}} ; x\right)=x^{2}
$$

This proves the theorem.

We recall that

$$
\begin{aligned}
& \mathcal{X}_{\left(1+x^{2}\right)}[0, \infty)=\left\{g:|g(x)| \leqq \mathcal{C}_{g}\left(1+x^{2}\right)\right\}, \\
& \mathcal{Y}_{\left(1+x^{2}\right)}[0, \infty)=\left\{g: g \in C[0, \infty) \cap \mathcal{X}_{\left(1+x^{2}\right)}[0, \infty)\right\}, \\
& \mathcal{Y}_{\left(1+x^{2}\right)}^{\sigma}[0, \infty)=\left\{g: g \in \mathcal{Y}_{\left(1+x^{2}\right)}[0, \infty) \text { such that } \lim _{x \rightarrow \infty} \frac{g(x)}{1+x^{2}}=\sigma\right\},
\end{aligned}
$$

where $\sigma$ is positive constant and $\mathcal{C}_{g}$ is a constant depends upon $g$.

Theorem 3.2 For all $g \in \mathcal{Y}_{\left(1+x^{2}\right)}^{\sigma}[0, \infty)$, we have

$$
\lim _{m \rightarrow \infty}\left\|\mathcal{S}_{m, q_{m}}^{*}\left(g ; \alpha_{[m]_{q_{m}}}, \beta_{[m]_{q_{m}}}\right)-g\right\|_{\left(1+x^{2}\right)}=0 .
$$

Proof Take the test function $g(t)=t^{p}$ for $p=0,1,2$ and use Lemma 2.2. From the Korovkin theorem we know, for every $g(t) \in \mathcal{Y}_{\left(1+x^{2}\right)}^{\sigma}[0, \infty), \mathcal{S}_{m, q_{m}}^{*}\left(t^{p} ; \alpha_{[m]_{q_{m}}}, \beta_{[m]]_{q_{m}}} ; x\right) \rightarrow x^{p}$ uniformly on $[0, \infty)$, as $m \rightarrow \infty$. When $\mathcal{S}_{m, q_{m}}^{*}\left(1 ; \alpha_{[m] q_{m}}, \beta_{[m]_{q m}} ; x\right)=1$, then clearly

$$
\lim _{m \rightarrow \infty}\left\|\mathcal{S}_{m, q_{m}}^{*}\left(1 ; \alpha_{[m] q_{m}}, \beta_{[m]_{q_{m}}}\right)-1\right\|_{\left(1+x^{2}\right)}=0
$$

In the case of $g(t)=t^{2}$

$$
\begin{aligned}
\left\|\mathcal{S}_{m, q_{m}}^{*}\left(t ; \alpha_{[m]_{q m}}, \beta_{[m]_{q_{m}}}\right)-x\right\|_{\left(1+x^{2}\right)} & =\sup _{x \geqq 0} \frac{\left|\mathcal{S}_{m, q_{m}}^{*}\left(t ; \alpha_{[m]_{q m}}, \beta_{[m]_{q_{m}}} ; x\right)-x\right|}{1+x^{2}} \\
& =\left(\frac{\alpha_{[m]_{q}}}{\beta_{[m]_{q}}}-1\right) \sup _{x \in[0, \infty)} \frac{x}{1+x^{2}}+\frac{1}{q_{m} \beta_{q_{m}}} \sup _{x \in[0, \infty)} \frac{1}{1+x^{2}} .
\end{aligned}
$$

Clearly, in the view of the results by (2.1), we see that

$$
\lim _{m \rightarrow \infty}\left\|\mathcal{S}_{m, q_{m}}^{*}\left(t ; \alpha_{[m]_{q m}}, \beta_{[m] q_{m}}\right)-x\right\|_{\left(1+x^{2}\right)}=0
$$


Similarly,

$$
\begin{aligned}
\left\|\mathcal{S}_{m, q_{m}}^{*}\left(t^{2} ; \alpha_{[m]_{q m}}, \beta_{[m]]_{q_{m}}}\right)-x^{2}\right\|_{\left(1+x^{2}\right)}= & \sup _{x \geqq 0} \frac{\left|\mathcal{S}_{m, q_{m}}^{*}\left(t^{2} ; \alpha_{[m]_{q_{m}}}, \beta_{[m]_{q m}} ; x\right)-x^{2}\right|}{1+x^{2}} \\
= & \left(\frac{\alpha_{[m]]_{m}}}{\beta_{[m]]_{m}}}-1\right)^{2} \sup _{x \geqq 0} \frac{x^{2}}{1+x^{2}} \\
& +\frac{\alpha_{[m]_{q_{m}}}}{\left(\beta_{[m]_{q_{m}}}\right)^{2}}\left(\frac{1+2 q_{m}}{q_{m}^{2}}+[1+2 \kappa]_{q_{m}}\right) \sup _{x \geqq 0} \frac{x}{1+x^{2}} \\
& +\frac{\left(1+q_{m}\right)}{q_{m}^{3}\left(\beta_{[m]_{q_{m}}}\right)^{2}} \sup _{x \geqq 0} \frac{1}{1+x^{2}} .
\end{aligned}
$$

Thus,

$$
\lim _{m \rightarrow \infty}\left\|\mathcal{S}_{m, q_{m}}^{*}\left(t^{2} ; \alpha_{[m]_{q m}}, \beta_{[m]_{q_{m}}}\right)-x^{2}\right\|_{\sigma}=0
$$

This completes the proof of Theorem 3.2.

\section{Order of approximation}

Let $g \in C_{H}[0, \infty)$ denote the set of all continuous functions on $[0, \infty)$ satisfying $|g(x)| \leqq$ $a e^{b x}$ for all $x \in[0, \infty)$ and where $a, b$ are positive constants. For a given $\delta^{*}>0$, the modulus of continuity of the function $g$ is defined as

$$
\omega^{*}\left(g ; \delta^{*}\right)=\sup _{\left|x_{1}-x_{2}\right| \leqq \delta^{*}, x_{1}, x_{2} \in[0, \infty)}\left|g\left(x_{1}\right)-g\left(x_{2}\right)\right| .
$$

Note that

$$
\left|g\left(x_{1}\right)-g\left(x_{2}\right)\right| \leqq\left(\frac{\left|x_{1}-x_{2}\right|}{\delta^{*}}+1\right) \omega^{*}\left(g ; \delta^{*}\right) .
$$

For all $g \in C_{B}[0, \infty)$, the modulus of continuity of order two is defined by

$$
\omega_{2}^{*}\left(g ; \delta^{*}\right)=\sup _{0<t \leqq \delta^{*}, y \in[0, \infty)}\|g(y+2 t)-2 g(y+t)+g(y)\|_{C_{B}[0, \infty)}
$$

where $C_{B}[0, \infty)$ is defined as a class of all real valued functions on $[0, \infty)$ which are bounded and uniformly continuous with the sup norm defined as $\|g\|_{C_{B}[0, \infty)}=$ $\sup _{x \geqq 0}|g(x)|$.

Theorem 4.1 For all $g \in C_{B}[0, \infty)$ and $q=q_{m}$ with the property $q_{m} \in(0,1)$, we have

$$
\begin{aligned}
& \left|\mathcal{S}_{m, q_{m}}^{*}\left(g ; \alpha_{[m]_{q_{m}}}, \beta_{[m]_{q_{m}}} ; x\right)-g(x)\right| \\
& \quad \leqq\left(1+\sqrt{\Theta_{m, q_{m}}(x)}\right) \omega^{*}\left(g ; \frac{1}{\sqrt{\beta_{[m]_{q_{m}}}}}\right),
\end{aligned}
$$

where $\Theta_{m, q_{m}}(x)=\frac{(1+q)}{q^{3}}+\frac{\alpha_{[m]_{q}}}{q^{2}}\left(1+2 q+q^{2}[1+2 \kappa]_{q}-2 q \beta_{[m]_{q}}\right) x+\left(\alpha_{[m]_{q}}-\beta_{[m]_{q}}\right)^{2} x^{2}$. 
Proof In the light of (4.1), (4.2) and the Cauchy-Schwarz inequality, we see that

$$
\begin{aligned}
& \left|\mathcal{S}_{m, q_{m}}^{*}\left(g ; \alpha_{[m]_{q_{m}}}, \beta_{[m]_{q_{m}}} ; x\right)-g(x)\right| \\
& \leqq \frac{\alpha_{[m]_{q_{m}}}}{e_{\kappa, q_{m}}\left(\alpha_{[m]_{q_{m}} x}\right)} \sum_{j=0}^{\infty} \mathcal{T}_{m, q_{m}}^{\kappa}(x) \int_{0}^{\infty / 1-q_{m}} \frac{e_{\kappa, q_{m}}\left(-[m]_{q_{m}} t\right)\left([m]_{q_{m}} t\right)^{\nabla_{j}}}{\left[\nabla_{j}\right]_{q} !}|g(t)-g(x)| \mathrm{d}_{q_{m}} t \\
& \leqq \frac{\alpha_{[m]_{q_{m}}}}{e_{\kappa, q_{m}}\left(\alpha_{\left.[m]_{q_{m}}\right)}\right)} \sum_{j=0}^{\infty} \mathcal{T}_{m, q_{m}}^{\kappa}(x) \\
& \times \int_{0}^{\infty / 1-q_{m}} \frac{e_{\kappa, q_{m}}\left(-\alpha_{[m]_{q_{m}}} t\right)\left(\alpha_{[m]_{q_{m}}} t\right)^{\nabla_{j}}}{\left[\nabla_{j}\right]_{q} !}\left(1+\frac{1}{\delta^{*}}|t-x|\right) \mathrm{d}_{q_{m}} t \omega^{*}\left(g ; \delta^{*}\right) \\
& =\left\{\frac { 1 } { \delta ^ { * } } \left(\frac{\alpha_{[m]_{q_{m}}}}{e_{\kappa, q_{m}}\left(\alpha_{[m]_{q_{m}} x}\right)} \sum_{j=0}^{\infty} \mathcal{T}_{m, q_{m}}^{\kappa}(x)\right.\right. \\
& \left.\left.\times \int_{0}^{\infty / 1-q_{m}} \frac{e_{\kappa, q_{m}}\left(\alpha_{[m]_{q_{m}} t}\right)\left(\alpha_{[m]_{q_{m}}} t\right)^{\nabla_{j}}}{\left[\nabla_{j}\right]_{q} !}(|t-x|) \mathrm{d}_{q_{m}} t\right)+1\right\} \omega^{*}\left(g ; \delta^{*}\right) \\
& \leq\left\{1+\frac{1}{\delta^{*}}\left[\frac{\alpha_{[m]_{q_{m}}}}{e_{\kappa, q_{m}}\left(\alpha_{[m]_{q_{m}}} x\right)} \sum_{j=0}^{\infty} \mathcal{T}_{m, q_{m}}^{\kappa}(x)\right.\right. \\
& \left.\left.\times \int_{0}^{\infty / 1-q_{m}} \frac{e_{\kappa, q_{m}}\left(-\alpha_{[m]_{q_{m}}} t\right)\left(\alpha_{[m]_{q m}} t\right)^{\nabla_{j}}}{\left[\nabla_{j}\right]_{q} !}\left(1+\frac{1}{\delta^{*}}(t-x)^{2}\right) \mathrm{d}_{q_{m}} t\right]^{\frac{1}{2}}\right\} \omega^{*}\left(g ; \delta^{*}\right) \\
& =\left\{1+\frac{1}{\delta^{*}}\left(\mathcal{S}_{m, q_{m}}^{*}\left((t-x)^{2} ; \alpha_{[m]_{q}}, \beta_{[m]} ; x\right)\right)^{\frac{1}{2}}\right\} \omega^{*}\left(g ; \delta^{*}\right) .
\end{aligned}
$$

If we take $\delta^{*}=\delta_{m, q_{m}}^{*}=\frac{1}{\sqrt{\beta_{[m] q_{m}}}}$, then we easily get the results.

Corollary 4.2 If we choose $\delta_{m, q_{m}}^{*}=\mathcal{S}_{m, q_{m}}^{*}\left((t-x)^{2} ; \alpha_{[m] q_{m}}, \beta_{[m]_{q_{m}}} ; x\right)$, then

$$
\left|\mathcal{S}_{m, q_{m}}^{*}\left(g ; \alpha_{[m]_{q_{m}}}, \beta_{[m]_{q_{m}}} ; x\right)-g(x)\right| \leqq 2 \omega^{*}\left(g ; \delta_{m, q_{m}}^{*}\right) .
$$

\section{Rate of convergence}

For all $g \in C[0, \infty)$ and $\lambda_{1}, \lambda_{2} \in[0, \infty)$, the set $\operatorname{Lip}_{\mathcal{C}}(\xi)$ is defined as

$$
\operatorname{Lip}_{\mathcal{C}}(\xi)=\left\{g:\left|g\left(\lambda_{1}\right)-g\left(\lambda_{2}\right)\right| \leqq \mathcal{C}\left|\lambda_{1}-\lambda_{2}\right|^{\xi}\right\}
$$

Moreover, for any $\chi \in C_{B}[0, \infty)$ one has the supremum norm

$$
\|\chi\|_{C_{B}[0, \infty)}=\sup _{x \geqq 0}|\chi(x)| .
$$

Let

$$
\begin{aligned}
& C_{B}^{2}[0, \infty)=\left\{\chi: \chi, \chi^{\prime}, \chi^{\prime \prime} \in C_{B}[0, \infty)\right\} \\
& \|\chi\|_{C_{B}^{2}[0, \infty)}=\|\chi\|_{C_{B}[0, \infty)}+\left\|\chi^{\prime}\right\|_{C_{B}[0, \infty)}+\left\|\chi^{\prime \prime}\right\|_{C_{B}[0, \infty)}
\end{aligned}
$$


Theorem 5.1 Let the sequences of positive numbers $\left\{\alpha_{[m] q_{m}}\right\}_{n \geqq 1}$ and $\left\{\beta_{[m] q_{m}}\right\}_{m \geqq 1}$ be defined by (2.1). Then, for each $g \in \operatorname{Lip}_{\mathcal{C}}(\xi)$ the operators $\mathcal{S}_{m, q_{m}}^{*}(\cdot ; \cdot)$ satisfy

$$
\begin{aligned}
& \left|\mathcal{S}_{m, q_{m}}^{*}\left(g ; \alpha_{[m] q_{m}}, \beta_{[m]_{q_{m}}} ; x\right)-g(x)\right| \\
& \quad \leq \mathcal{C}\left\{\frac{\left(1+q_{m}\right)}{q_{m}^{3}\left(\beta_{[m] q_{m}}\right)^{2}}+\frac{\alpha_{[m]_{q_{m}}}}{\left(q \beta_{[m]_{q_{m}}}\right)^{2}}\left(1+2 q_{m}+q_{m}^{2}[1+2 \kappa]_{q_{m}}-2 q_{m} \beta_{[m]]_{m}}\right) x\right. \\
& \left.\quad+\left(\frac{\alpha_{[m] q_{m}}}{\beta_{[m] q_{m}}}-1\right)^{2} x^{2}\right\}^{\frac{\xi}{2}} .
\end{aligned}
$$

Proof We use the result by (5.1):

$$
\begin{aligned}
\left|\mathcal{S}_{m, q_{m}}^{*}\left(g ; \alpha_{[m] q_{m}}, \beta_{[m] q_{m}} ; x\right)-g(x)\right| & \leq\left|\mathcal{S}_{m, q_{m}}^{*}\left(g(t)-g(x) ; \alpha_{[m]_{q_{m}}}, \beta_{[m]]_{m}} ; x\right)\right| \\
& \leq \mathcal{S}_{m, q_{m}}^{*}\left(|g(t)-g(x)| ; \alpha_{[m]_{q_{m}}}, \beta_{[m]_{q_{m}}} ; x\right) \\
& \leq \mathcal{C} \mathcal{S}_{m, q_{m}}^{*}\left(|t-x|^{\xi} ; x\right) .
\end{aligned}
$$

Therefore,

$$
\begin{aligned}
&\left|\mathcal{S}_{m, q_{m}}^{*}\left(g ; \alpha_{[m]_{q_{m}}}, \beta_{[m]_{q_{m}}} ; x\right)-g(x)\right| \leq \mathcal{C} \frac{\alpha_{[m]_{m}}}{e_{\kappa, q_{m}}\left(\alpha_{[m] q_{m}}\right)} \sum_{j=0}^{\infty} \mathcal{T}_{m, q_{m}}^{\kappa}(x) \int_{0}^{\infty / 1-q_{m}} \frac{e_{\kappa, q_{m}}\left(-\alpha_{[m] q_{m}} t\right)\left(\alpha_{[m] q_{m}} t\right)^{\nabla_{j}}}{\left[\nabla_{j}\right]_{q} !}|t-x| \mathrm{d}_{q_{m}} t \\
& \leqq \mathcal{C} \frac{\alpha_{[m] q_{m}}}{e_{\kappa, q_{m}}\left(\alpha_{[m]_{q_{m}}}\right)} \sum_{j=0}^{\infty}\left(\mathcal{T}_{m, q_{m}}^{\kappa}(x)\right)^{\frac{2-\xi}{2}}\left(\mathcal{T}_{m, q_{m}}^{\kappa}(x)\right)^{\frac{\xi}{2}} \\
& \times \int_{0}^{\infty / 1-q_{m}} \frac{e_{q_{m}}\left(-\alpha_{[m]_{q_{m}}} t\right)\left(\alpha_{[m] q_{m}} t\right)^{\nabla_{j}}}{\left[\nabla_{j}\right]_{q} !}|t-x| \mathrm{d}_{q_{m}} t \\
& \leqq \mathcal{C}\left(\frac{\alpha_{[m]_{q_{m}}}}{e_{\kappa, q_{m}}\left(\alpha_{[m]_{q_{m}}} x\right)} \sum_{j=0}^{\infty} \mathcal{T}_{m, q_{m}}^{\kappa}(x) \int_{0}^{\infty / 1-q_{m}} \frac{e_{q_{m}}\left(-\alpha_{[m]_{q_{m}}} t\right)\left(\alpha_{[m]_{q_{m}}} t\right)^{\nabla_{j}}}{\left[\nabla_{j}\right]_{q} !} \mathrm{d}_{q_{m}} t\right)^{\frac{2-\xi}{2}} \\
& \quad \times\left(\frac{\alpha_{[m]_{q_{m}}}}{e_{\kappa, q_{m}}\left(\alpha_{[m]_{q_{m}}} x\right)} \sum_{j=0}^{\infty} \mathcal{T}_{m, q_{m}}^{\kappa}(x) \int_{0}^{\infty / 1-q_{m}} \frac{e_{q_{m}}\left(-\alpha_{[m]_{q_{m}}} t\right)\left(\alpha_{[m] q_{m}} t\right)^{\nabla_{j}}}{\left[\nabla_{j}\right]_{q} !}|t-x|^{2} \mathrm{~d}_{q_{m}} t\right)^{\frac{\xi}{2}} \\
&=\mathcal{C}\left(\mathcal{S}_{m, q_{m}}^{*}(t-x)^{2} ; \alpha_{[m]]_{m}}, \beta_{[m]_{q_{m}}} ; x\right)^{\frac{\xi}{2}} .
\end{aligned}
$$

This completes the proof.

Theorem 5.2 For all $\chi \in C_{B}^{2}[0, \infty)$ defined by (5.3), we have

$$
\left|\mathcal{S}_{m, q_{m}}^{*}\left(\chi ; \alpha_{[m]_{q_{m}}}, \beta_{[m]_{q_{m}}} ; x\right)-\chi(x)\right| \leqq\left(\frac{\Lambda_{m, q_{m}}(x)}{2}+\Theta_{m, q_{m}}(x)\right)\|\chi\|_{C_{B}^{2}[0, \infty)},
$$

where $\Lambda_{m, q_{m}}(x)=\frac{\left(1+q_{m}\right)}{q_{m}^{3}\left(\beta_{[m] q_{m}}\right)^{2}}+\frac{\alpha_{[m] q_{m}}}{\left(q_{m} \beta_{[m]} q_{m}\right)^{2}}\left(1+2 q_{m}+q_{m}^{2}[1+2 \kappa]_{q_{m}}-2 q_{m} \beta_{[m] q_{m}}\right) x+\left(\frac{\alpha_{[m]} q_{m}}{\beta_{[m] q_{m}}}-\right.$ $1)^{2} x^{2}$ and $\Theta_{m, q_{m}}(x)=\left(\frac{\alpha_{[m] q_{m}}}{\beta_{[m] q_{m}}}-1\right) x+\frac{1}{q \beta_{[m] q_{m}}}$. 
Proof Take $\chi \in C_{B}^{2}[0, \infty)$ and $\phi \in(x, t)$. On applying the linearity on generalized mean value theorem of Taylor series, we conclude that

$$
\begin{aligned}
\mathcal{S}_{m, q_{m}}^{*}\left(\chi ; \alpha_{[m]_{q m}}, \beta_{[m]_{q_{m}}} ; x\right)-\chi(x)= & \chi^{\prime}(x) \mathcal{S}_{m, q_{m}}^{*}\left((t-x) ; \alpha_{[m]_{q_{m}}}, \beta_{[m]_{q_{m}}} ; x\right) \\
& +\frac{\chi^{\prime \prime}(\phi)}{2} \mathcal{S}_{m, q_{m}}^{*}\left((t-x)^{2} ; ; \alpha_{[m]_{q_{m}}}, \beta_{[m]_{q_{m}}} ; x\right) .
\end{aligned}
$$

Therefore,

$$
\begin{aligned}
& \left|\mathcal{S}_{m, q_{m}}^{*}\left(\chi ; \alpha_{[m]_{q_{m}}}, \beta_{[m]_{q_{m}}} ; x\right)-\chi(x)\right| \\
& \leqq \\
& \leq\left\{\left(\frac{\alpha_{[m]_{q}}}{\beta_{[m]_{q}}}-1\right) x+\frac{1}{q \beta_{[m]_{q}}}\right\}\left\|\chi^{\prime}\right\|_{C_{B}[0, \infty)} \\
& \quad+\left\{\frac{\left(1+q_{m}\right)}{q_{m}^{3}\left(\beta_{[m]_{q m}}\right)^{2}}+\frac{\alpha_{[m]_{q_{m}}}}{\left(q_{m} \beta_{[m]_{q_{m}}}\right)^{2}}\left(1+2 q_{m}+q_{m}^{2}[1+2 \kappa]_{q_{m}}-2 q_{m} \beta_{[m]_{q_{m}}}\right) x\right. \\
& \left.\quad+\left(\frac{\alpha_{[m]_{q_{m}}}}{\beta_{[m]_{m}}}-1\right)^{2} x^{2}\right\} \frac{\left\|\chi^{\prime \prime}\right\|_{C_{B}[0, \infty)}}{2} .
\end{aligned}
$$

From (5.4) we easily see that

$$
\begin{aligned}
& \left|\mathcal{S}_{m, q_{m}}^{*}\left(\chi ; \alpha_{[m]_{q_{m}}}, \beta_{[m]_{q_{m}}} ; x\right)-\chi(x)\right| \\
& \leq\left\{\left(\frac{\alpha_{[m]_{q_{m}}}}{\beta_{[m]_{q_{m}}}}-1\right) x+\frac{1}{q_{m} \beta_{[m]_{q_{m}}}}\right\}\|\chi\|_{C_{B}^{2}[0, \infty)} \\
& \quad+\left\{\frac{\left(1+q_{m}\right)}{q_{m}^{3}\left(\beta_{[m]_{q_{m}}}\right)^{2}}+\frac{\alpha_{[m]_{q_{m}}}}{\left(q_{m} \beta_{[m]_{q_{m}}}\right)^{2}}\left(1+2 q_{m}+q_{m}^{2}[1+2 \kappa]_{q_{m}}-2 q_{m} \beta_{[m]_{q_{m}}}\right) x\right. \\
& \left.\quad+\left(\frac{\alpha_{[m]_{q_{m}}}}{\beta_{[m]_{q_{m}}}}-1\right)^{2} x^{2}\right\} \frac{\|\chi\|_{C_{B}^{2}[0, \infty)}}{2} .
\end{aligned}
$$

This completes the proof.

\section{Some direct theorem}

Let $g$ and $\Phi \in C_{B}^{2}[0, \infty)$. For a given $\delta^{*}>0$, the Peetre's $K$-functional [41] is defined as

$$
\begin{aligned}
& K\left(g ; \delta^{*}\right)=\inf \left\{\|g-\Phi\|_{C_{B}[0, \infty)}+\delta^{*}\|\Phi\|_{C_{B}^{2}[0, \infty)}\right\}, \\
& K\left(g ; \delta^{*}\right) \leqq \mathcal{W} \omega_{2}^{*}\left(g ;\left(\delta^{*}\right)^{\frac{1}{2}}\right),
\end{aligned}
$$

where $\omega_{2}^{*}$ is the modulus of continuity of order two defined in (4.3). By [14], there exists an absolute constant $\mathcal{C}>0$ such that

$$
K\left(g ; \delta^{*}\right) \leqq \mathcal{C}\left\{\omega_{2}^{*}\left(g ; \sqrt{\delta^{*}}\right)+\min \left(1, \delta^{*}\right)\|g\|\right\}
$$

Moreover, in the spaces of weighted modulus of continuity for each arbitrary $g \in$ $\mathcal{Y}_{\left(1+x^{2}\right)}^{\sigma}[0, \infty)$ we have [10]

$$
\Omega^{*}\left(g ; \delta^{*}\right)=\sup _{x \geqq 0,|u| \leqq \delta^{*}} \frac{|g(x+u)-g(x)|}{\left(1+u^{2}\right)\left(1+x^{2}\right)},
$$




$$
\begin{aligned}
& \lim _{\delta \rightarrow 0} \Omega^{*}\left(g ; \delta^{*}\right)=0 \\
& |g(t)-g(x)| \leqq 2\left(1+\frac{|t-x|}{\delta^{*}}\right)\left(1+\left(\delta^{*}\right)^{2}\right)\left(1+x^{2}\right)\left(1+(t-x)^{2}\right) \Omega^{*}\left(g ; \delta^{*}\right),
\end{aligned}
$$

where $t, x \in[0, \infty)$.

Theorem 6.1 For all $g \in C_{B}[0, \infty)$ and $q=q_{m}$ with the number $q_{m} \in(0,1)$, we have

$$
\begin{aligned}
& \left|\mathcal{S}_{m, q_{m}}^{*}\left(g ; \alpha_{[m]_{q_{m}}}, \beta_{[m]_{q_{m}}} ; x\right)-g(x)\right| \\
& \quad \leqq 2 \mathcal{C}\left\{\omega_{2}^{*}\left(g ; \sqrt{\frac{\Lambda_{m, q_{m}}(x)}{4}+\frac{\Theta_{m, q_{m}}(x)}{2}}\right)+\min \left(1, \frac{\Lambda_{m, q_{m}}(x)}{4}+\frac{\Theta_{m, q_{m}}(x)}{2}\right)\|g\|_{C_{B}[0, \infty)}\right\},
\end{aligned}
$$

where $\mathcal{C}$ is an absolute positive constant.

Proof Let $\Phi \in C_{B}[0, \infty)$. Applying Theorem 5.2, we get the result asserted by Theorem 6.1. Therefore,

$$
\begin{aligned}
& \left|\mathcal{S}_{m, q_{m}}^{*}\left(g ; \alpha_{[m]_{q_{m}}}, \beta_{[m]_{q_{m}}} ; x\right)-g(x)\right| \\
& \leqq\left|\mathcal{S}_{m, q_{m}}^{*}\left((g-\Phi) ; \alpha_{[m]_{q_{m}}}, \beta_{[m]_{q_{m}}} ; x\right)\right| \\
& +\left|\mathcal{S}_{m, q_{m}}^{*}\left(\Phi ; \alpha_{[m]_{q m}}, \beta_{[m]_{q_{m}}} ; x\right)-\Phi(x)\right|+|g(x)-\Phi(x)| \\
& \leqq 2\|g-\Phi\|_{C_{B}[0, \infty)}+\left(\frac{\Lambda_{m, q_{m}}(x)}{2}+\Theta_{m, q_{m}}(x)\right)\|\Phi\|_{C_{B}^{2}[0, \infty)} \\
& =2\left(\|g-\Phi\|_{C_{B}[0, \infty)}+\frac{\Lambda_{m, q_{m}}(x)}{4}+\frac{\Theta_{m, q_{m}}(x)}{2}\|\Phi\|_{C_{B}^{2}[0, \infty)}\right) .
\end{aligned}
$$

On taking the infimum over all $\Phi \in C_{B}^{2}[0, \infty)$ and applying the result (6.2), we get

$$
\left|\mathcal{S}_{m, q_{m}}^{*}\left(g ; \alpha_{[m]_{q_{m}}}, \beta_{[m]_{q_{m}}} ; x\right)-g(x)\right| \leqq 2 K\left(g ; \frac{\Lambda_{m, q_{m}}(x)}{4}+\frac{\Theta_{m, q_{m}}(x)}{2}\right)
$$

Therefore, from (6.3), we get an absolute constant $\mathcal{C}>0$ such that the result holds.

Theorem 6.2 For each $g \in \mathcal{Y}_{\left(1+x^{2}\right)}^{\sigma}[0, \infty)$,

$$
\sup _{x \in\left[0, \Psi_{\kappa, q_{m}}(m)\right)} \frac{\left|\mathcal{S}_{m, q_{m}}^{*}\left(g ; \alpha_{[m]_{q_{m}}}, \beta_{[m]_{q_{m}}} ; x\right)-g(x)\right|}{1+x^{2}} \leqq \mathcal{N}\left(1+\Psi_{\kappa, q_{m}}(m)\right) \Omega^{*}\left(g ; \sqrt{\Psi_{\kappa, q_{m}}}\right)
$$

where $\mathcal{N}=2\left(1+\mathcal{N}_{1}+2 \sqrt{2} \mathcal{N}_{2}\right)$ and $\Psi_{\kappa, q_{m}}(m)=\max \left\{\gamma_{1, n_{q_{m}}}, \gamma_{2, n_{q m}}, \gamma_{3, n_{q_{m}}}\right\}$ with $\gamma_{1, n_{q_{m}}}=$ $\frac{\left(1+q_{m}\right)}{q_{m}^{3}\left(\beta_{[m]_{m}}\right)^{2}}, \gamma_{2, n_{q_{m}}}=\frac{\alpha_{\left[m q_{q_{m}}\right.}}{\left(q_{m} \beta_{[m] q_{m}}\right)^{2}}\left(1+2 q_{m}+q_{m}^{2}[1+2 \kappa]_{q_{m}}-2 q_{m} \beta_{[m]_{q_{m}}}\right)$ and $\gamma_{3, n_{q_{m}}}=\left(\frac{\alpha_{[m] q_{m}}}{\beta_{\left[m q_{m}\right.}}-1\right)^{2}$.

Proof From (6.6), we have

$$
\begin{aligned}
& \left|\mathcal{S}_{m, q_{m}}^{*}\left(g ; \alpha_{[m]_{q_{m}}}, \beta_{[m]_{q_{m}}} ; x\right)-g(x)\right| \\
& \quad \leqq 2\left(1+\left(\delta^{*}\right)^{2}\right)\left(1+x^{2}\right) \Omega^{*}\left(g ; \delta^{*}\right)\left(1+\mathcal{S}_{m, q_{m}}^{*}\left((t-x)^{2} ; \alpha_{[m]_{q_{m}}}, \beta_{[m]_{q_{m}}} ; x\right)\right.
\end{aligned}
$$


Alotaibi Journal of Inequalities and Applications

(2020)

2020:121

Page 13 of 15

$$
\left.+\mathcal{S}_{m, q_{m}}^{*}\left(\left(1+(t-x)^{2}\right) \frac{|t-x|}{\delta^{*}} ; \alpha_{[m]_{q m}}, \beta_{[m]_{q m}} ; x\right)\right) .
$$

From the Cauchy-Schwartz inequality obviously

$$
\begin{aligned}
\mathcal{S}_{m, q_{m}}^{*} & \left(\left(1+(t-x)^{2}\right) \frac{|t-x|}{\delta^{*}} ; \alpha_{[m]_{q m}}, \beta_{[m]_{q m}} ; x\right) \\
\leqq & 2\left(\mathcal{S}_{m, q_{m}}^{*}\left(1+(t-x)^{4} ; \alpha_{[m]_{q m}}, \beta_{[m]_{q_{m}}} ; x\right)\right)^{\frac{1}{2}} \\
& \times\left(\mathcal{S}_{m, q_{m}}^{*}\left(\frac{(t-x)^{2}}{\left(\delta^{*}\right)^{2}} ; \alpha_{[m]_{q m}}, \beta_{[m]_{q_{m}}} ; x\right)\right)^{\frac{1}{2}} .
\end{aligned}
$$

From Lemma 2.3, we easily conclude that

$$
\mathcal{S}_{m, q_{m}}^{*}\left((t-x)^{2} ; \alpha_{[m]_{q m}}, \beta_{[m]_{q m}} ; x\right) \leqq \Psi_{\kappa, q_{m}}(m)\left(1+x+x^{2}\right),
$$

where

$$
\begin{aligned}
\Psi_{\kappa, q_{m}}(m)= & \max \left\{\frac{\left(1+q_{m}\right)}{q_{m}^{3}\left(\beta_{[m]_{q m}}\right)^{2}}, \frac{\alpha_{[m]_{q_{m}}}}{\left(q_{m} \beta_{[m]_{q_{m}}}\right)^{2}}\left(1+2 q_{m}+q_{m}^{2}[1+2 \kappa]_{q_{m}}-2 q_{m} \beta_{[m]_{q m}}\right),\right. \\
& \left.\left(\frac{\alpha_{[m]_{q_{m}}}}{\beta_{[m]_{q_{m}}}}-1\right)^{2}\right\} .
\end{aligned}
$$

There exists a positive constant $\mathcal{C}_{1}$ satisfying

$$
\mathcal{S}_{m, q_{m}}^{*}\left((t-x)^{2} ; \alpha_{[m]_{q m}}, \beta_{[m]_{q_{m}}} ; x\right) \leqq \mathcal{N}_{1}\left(1+x+x^{2}\right)
$$

Similarly,

$$
\mathcal{S}_{m, q_{m}}^{*}\left((t-x)^{4} ; \alpha_{[m]_{q_{m}}}, \beta_{[m]_{q_{m}}} ; x\right) \leqq \varphi_{\kappa, q_{m}}(m)\left(1+x+x^{2}+x^{3}+x^{4}\right)
$$

where

$$
\begin{aligned}
\xi_{\kappa, q_{m}}(m) & \max \left\{\frac{\left(1+q_{m}\right)^{2}}{q_{m}^{6}\left(\beta_{[m]_{q}}\right)^{4}},\left[\frac{2\left(1+q_{m}\right) \alpha_{[m]_{q_{m}}}}{\left(q_{m}^{5} \beta_{[m]_{q_{m}}}\right)^{4}}\left(1+2 q_{m}+q_{m}^{2}[1+2 \kappa]_{q_{m}}-2 q_{m} \beta_{[m] q_{m}}\right)\right]\right. \\
& {\left[\frac{\left(\alpha_{[m]_{q_{m}}}\right)^{2}}{\left(q_{m} \beta_{[m]_{q_{m}}}\right)^{4}}\left(1+2 q_{m}+q_{m}^{2}[1+2 \kappa]_{q_{m}}-2 q_{m} \beta_{[m]_{q_{m}}}\right)^{2}\right.} \\
& \left.+\frac{2\left(1+q_{m}\right)}{q_{m}^{3}\left(\beta_{[m]_{q_{m}}}\right)^{2}}\left(\frac{\alpha_{[m]_{q_{m}}}}{\beta_{[m]_{q_{m}}}}-1\right)^{2}\right] \\
& \frac{2 \alpha_{[m]_{q_{m}}}}{\left(q_{m} \beta_{[m]_{q_{m}}}\right)^{2}}\left(1+2 q_{m}+q_{m}^{2}[1+2 \kappa]_{q_{m}}-2 q_{m} \beta_{[m]_{q_{m}}}\right)\left(\frac{\alpha_{[m]_{q_{m}}}}{\beta_{[m]_{q_{m}}}}-1\right)^{2} \\
& \left.\left(\frac{\alpha_{[m]_{q_{m}}}}{\beta_{[m]}}-1\right)^{4}\right\} .
\end{aligned}
$$


Since $\left\{\alpha_{[m]_{q}}\right\}$ and $\left\{\beta_{[m]_{q}}\right\}$ are the sequences satisfying (2.1) and $\lim _{n \rightarrow \infty} q_{m}=1$, there exists a constant $\mathcal{N}_{2}>0$, such that

$$
\left(\mathcal{S}_{m, q_{m}}^{*}\left(1+(t-x)^{4} ; \alpha_{[m]_{q m}}, \beta_{[m] q_{m}} ; x\right)\right)^{\frac{1}{2}} \leqq \mathcal{N}_{2}\left(2+x+x^{2}+x^{3}+x^{4}\right)^{\frac{1}{2}}
$$

Clearly in the light of (6.7)-(6.11), we conclude that

$$
\left(\mathcal{S}_{m, q_{m}}^{*}\left(\frac{(t-x)^{2}}{\left(\delta^{*}\right)^{2}} ; \alpha_{[m]_{q_{m}}}, \beta_{[m]_{q_{m}}} ; x\right)\right)^{\frac{1}{2}} \leqq \frac{1}{\delta^{*}}\left(\Psi_{\kappa, q_{m}}(m)\right)^{\frac{1}{2}}\left(1+x+x^{2}\right)^{\frac{1}{2}}
$$

Thus by combining (6.8)-(6.12) in (6.7) and if we put $\delta^{*}=\sqrt{\Psi_{\kappa, q_{m}}(m)}$ and taking the supremum over all $x \in\left[0, \Psi_{\kappa, q_{m}}(m)\right)$, we get the result.

Remark 6.3 For future work, some convergence properties of operators through summability techniques (see [11, 12, 16, 20, 29-31]) can be examined.

\section{Acknowledgements}

This project was funded by the Deanship of Scientific Research (DSR) at King Abdulaziz University, Jeddah, under grant no. (RG-84-130-38). The author, therefore, acknowledges with thank DSR for technical and financial support.

\section{Funding}

This project was funded by the Deanship of Scientific Research (DSR) at King Abdulaziz University, Jeddah, under grant no. (RG-84-130-38).

Availability of data and materials

Not applicable.

\section{Competing interests}

The author declares he has no competing interests.

\section{Authors' contributions}

The author read and approved the final manuscript.

\section{Publisher's Note}

Springer Nature remains neutral with regard to jurisdictional claims in published maps and institutional affiliations.

Received: 4 December 2019 Accepted: 16 April 2020 Published online: 01 May 2020

\section{References}

1. Acar, T.: Quantitative $q$-Voronovskaya and $q$-Grüss-Voronovskaya-type results for $q$-Szász operators. Georgian Math. J. 23, 459-468 (2016)

2. Acar, T., Aral, A.: On pointwise convergence of $q$-Bernstein operators and their $q$-derivatives. Numer. Funct. Anal. Optim. 36, 287-304 (2015)

3. Acar, T., Aral, A., Mohiuddine, S.A.: On Kantorovich modification of (p, q)-Baskakov operators. J. Inequal. Appl. 2016 Article ID 98 (2016)

4. Acar, T., Aral, A., Mohiuddine, S.A.: Approximation by bivariate $(p, q)$-Bernstein-Kantorovich operators. Iran. J. Sci. Technol., Trans. A, Sci. 42, 655-662 (2018)

5. Acar, T., Aral, A., Mohiuddine, S.A.: On Kantorovich modification of (p, q)-Bernstein operators. Iran. J. Sci. Technol, Trans. A, Sci. 42, 1459-1464 (2018)

6. Acar, T., Aral, A., Rasa, l.: Positive linear operators preserving $\tau$ and $\tau^{2}$. Constr. Math. Anal. 2(3), 98-102 (2019)

7. Acar, T., Mohiuddine, S.A., Mursaleen, M.: Approximation by $(p, q)$-Baskakov-Durrmeyer-Stancu operators. Complex Anal. Oper. Theory 12, 1453-1468 (2018)

8. Alotaibi, A., Nasiruzzaman, M., Mursaleen, M.: A Dunkl type generalization of Szász operators via post-quantum calculus. J. Inequal. Appl. 2018, Article ID 287 (2018)

9. Altomare, F.: Korovkin type theorems and approximation by positive linear operators. Surv. Approx. Theory 5, 92-164 (2010)

10. Atakut, Ç., Ispir, N.: Approximation by modified Szász-Mirakjan operators on weighted spaces. Proc. Indian Acad. Sci. Math. Sci. 112, 571-578 (2002)

11. Belen, C., Mohiuddine, S.A.: Generalized weighted statistical convergence and application. Appl. Math. Comput. 219, 9821-9826 (2013)

12. Braha, N.L., Srivastava, H.M., Mohiuddine, S.A.: A Korovkin's type approximation theorem for periodic functions via the statistical summability of the generalized de la Vallée Poussin mean. Appl. Math. Comput. 228, 162-169 (2014) 
13. Cheikh, B., Gaied, Y., Zaghouani, M.: A q-Dunkl-classical q-Hermite type polynomials. Georgian Math. J. 21, 125-137 (2014)

14. Ciupa, A.: A class of integral Favard-Szász type operators. Stud. Univ. Babeş-Bolyai, Math. 40, 39-47 (1995)

15. De Sole, A., Kac, V.G.: On integral representation of q-gamma and q-beta functions. Atti Accad. Naz. Lincei, Cl. Sci. Fis. Mat. Nat. Rend. Lincei (9), Mat. Appl. 16, 11-29 (2005)

16. Edely, O.H.H., Mohiuddine, S.A., Noman, A.K.: Korovkin type approximation theorems obtained through generalized statistical convergence. Appl. Math. Lett. 23(11), 1382-1387 (2010)

17. Iç̧̋zz, G., Çekim, B.: Dunkl generalization of Szász operators via q-calculus. J. Inequal. Appl. 2015, Article ID 284 (2015)

18. Ince Ilarslan, H.G., Acar, T.: Approximation by bivariate $(p, q)$-Baskakov-Kantorovich operators. Georgian Math. J. 25(3), 397-407 (2018)

19. Jackson, F.H.: On q-definite integrals. Q. J. Pure Appl. Math. 15, 193-203 (1910)

20. Kadak, U., Mohiuddine, S.A.: Generalized statistically almost convergence based on the difference operator which includes the $(p, q)$-gamma function and related approximation theorems. Results Math. 73(1), Article ID 9 (2018)

21. Kajla, A., Acar, T.: Modified $\alpha$-Bernstein operators with better approximation properties. Ann. Funct. Anal. 10(4), 570-582 (2019)

22. Lupaş, A.: A q-analogue of the Bernstein operator. Univ. Cluj-Napoca Semin. Numer. Stat. Calc. 9, 85-92 (1987)

23. Maurya, R., Sharma, H., Gupta, C.: Approximation properties of Kantorovich type modifications of (p,q)-Meyer-König-Zeller operators. Constr. Math. Anal. 1(1), 58-72 (2018)

24. May, C.P.: On Phillips operators. J. Approx. Theory 20, 315-322 (1977)

25. Milovanović, G.V., Mursaleen, M., Nasiruzzaman, M.: Modified Stancu type Dunkl generalization of Szász-Kantorovich operators. Rev. R. Acad. Cienc. Exactas Fís. Nat., Ser. A Mat. 112(1), 135-151 (2018)

26. Mohiuddine, S.A., Acar, T., Alghamdi, M.A.: Genuine modified Bernstein-Durrmeyer operators. J. Inequal. Appl. 2018 Article ID 104 (2018)

27. Mohiuddine, S.A., Acar, T., Alotaibi, A.: Construction of a new family of Bernstein-Kantorovich operators. Math. Methods Appl. Sci. 40, 7749-7759 (2017)

28. Mohiuddine, S.A., Acar, T., Alotaibi, A.: Durrmeyer type $(p, q)$-Baskakov operators preserving linear functions. J. Math. Inequal. 12(4), 961-973 (2018)

29. Mohiuddine, S.A., Alamri, B.A.S.: Generalization of equi-statistical convergence via weighted lacunary sequence with associated Korovkin and Voronovskaya type approximation theorems. Rev. R. Acad. Cienc. Exactas Fís. Nat., Ser. A Mat. 113(3), 1955-1973 (2019)

30. Mohiuddine, S.A., Asiri, A., Hazarika, B.: Weighted statistical convergence through difference operator of sequences of fuzzy numbers with application to fuzzy approximation theorems. Int. J. Gen. Syst. 48(5), 492-506 (2019)

31. Mohiuddine, S.A., Hazarika, B., Alghamdi, M.A.: Ideal relatively uniform convergence with Korovkin and Voronovskaya types approximation theorems. Filomat 33(14), 4549-4560 (2019)

32. Mohiuddine, S.A., Özger, F.: Approximation of functions by Stancu variant of Bernstein-Kantorovich operators based on shape parameter $\alpha$. Rev. R. Acad. Cienc. Exactas Fís. Nat., Ser. A Mat. 114(2), Article 70 (2020)

33. Mursaleen, M., Ansari, K.J., Khan, A.: On (p, q)-analogue of Bernstein operators. Appl. Math. Comput. 266, 874-882 (2015) [Erratum: Appl. Math. Comput. 278, 70-71 (2016)]

34. Mursaleen, M., Ansari, K.J., Khan, A.: Some approximation results by $(p, q)$-analogue of Bernstein-Stancu operators Appl. Math. Comput. 264, 392-402 (2015) [Corrigendum: Appl. Math. Comput. 269, 744-746 (2015)]

35. Mursaleen, M., Nasiruzzaman, M., Alotaibi, A.: On modified Dunkl generalization of Szász operators via q-calculus. J. Inequal. Appl. 2017, Article ID 38 (2017)

36. Nasiruzzaman, M., Mukheimer, A., Mursaleen, M.: A Dunkl type generalization of Szasz-Kantorovich operators via post-quantum calculus. Symmetry 11, Article ID 232 (2019)

37. Nasiruzzaman, M., Mukheimer, A., Mursaleen, M.: Approximation results on Dunkl generalization of Phillips operators via q-calculus. Adv. Differ. Equ. 2019, Article ID 244 (2019)

38. Nasiruzzaman, M., Rao, N.: A generalized Dunkl type modifications of Phillips-operators. J. Inequal. Appl. 2018, Article ID 323 (2018)

39. Nasiruzzaman, M., Rao, N.: A generalized Dunkl type modification of Phillips-operators. J. Inequal. Appl. 2018, Article ID 323 (2018)

40. Nasiruzzaman, M., Rao, N., Wazir, S., Kumar, R.: Approximation on parametric extension of Baskakov Durrmeyer operators on weighted spaces. J. Inequal. Appl. 2019, Article ID 103 (2019)

41. Peetre, J.: A theory of interpolation of normed spaces, Notas de Matemática, Rio de Janeiro, Instituto de Matemática Pura e Aplicada, Conselho Nacional de Pesquisas (1968)

42. Rao, N., Wafi, A., Acu, A.M.: q-Szász-Durrmeyer type operators based on Dunkl analogue. Complex Anal. Oper. Theory 13, 915-934 (2019)

43. Rosenblum, M.: Generalized Hermite polynomials and the Bose-like oscillator calculus. Oper. Theory, Adv. Appl. 73, 369-396 (1994)

44. Srivastava, H.M., Mursaleen, M., Alotaibi, A., Nasiruzzaman, M., Al-Abied, A.: Some approximation results involving the q-Szasz-Mirakjan-Kantorovich type operators via Dunkl's generalization. Math. Methods Appl. Sci. 40, 5437-5452 (2017)

45. Srivastava, H.M., Mursaleen, M., Nasiruzzaman, M.: Approximation by a class of $q$-beta operators of the second kind via the Dunkl-type generalization on weighted spaces. Complex Anal. Oper. Theory 13(3), 1537-1556 (2019)

46. Srivastava, H.M., Özger, F., Mohiuddine, S.A.: Construction of Stancu type Bernstein operators based on Bézier bases with shape parameter $\lambda$. Symmetry 11(3), Article ID 316 (2019)

47. Sucu, S.: Dunkl analogue of Szász operators. Appl. Math. Comput. 244, 42-48 (2014)

48. Szász, O: Generalization of S. Bernstein's polynomials to the infinite interval. J. Res. Natl. Bur. Stand. 45, $239-245$ (1950)

49. Ulusoy, G., Acar, T.: q-Voronovskaya type theorems for q-Baskakov operators. Math. Methods Appl. Sci. 39(12), $3391-3401(2016)$ 